\section{Innovating healthcare delivery to address noncommunicable diseases in low-income settings: the example of hypertension}

\author{
Peter Piot ${ }^{1}$, Ann Aerts ${ }^{2}$, David A Wood ${ }^{3}$, Peter Lamptey ${ }^{4}$, Samuel Oti ${ }^{5}$, \\ Kenneth Connell ${ }^{6}$, Prabhakaran Dorairaj ${ }^{7}$, Jo I Boufford ${ }^{8}$, Aya Caldwell ${ }^{2}$ \\ \& Pablo Perel*,1
}

\begin{abstract}
London Dialogue event, The Hospital Club, 24 Endell St, London, WC2H 9HQ, London, UK, 1 December 2015
\end{abstract}

Hypertension is a global health issue causing almost 10 million deaths annually, with a disproportionate number occurring in low- and middle-income countries. The condition can be managed effectively, but there is a need for innovation in healthcare delivery to alleviate its burden. This paper presents a number of innovative delivery models from a number of different countries, including Kenya, Ghana, Barbados and India. These models were presented at the London Dialogue event, which was cohosted by the Novartis Foundation and the London School of Hygiene \& Tropical Medicine Centre for Global Noncommunicable Diseases on 1 December 2015. It is argued that these models are applicable not only to hypertension, but provide valuable lessons to address other noncommunicable diseases.

First draft submitted: 6 April 2016; Accepted for publication: 28 April 2016;

Published online: 13 June 2016

High blood pressure (BP) is the world's leading disease burden, responsible for almost 10 million deaths annually, which is equal to the number of deaths caused by all infectious diseases combined [1,2]. The majority of this mortality occurs in low- and middle-income countries (LMIC)s, where people are presenting with hypertension at a younger age [3] and experience worse outcomes than in high-income countries [4]. Moreover, the proportion of people with undiagnosed, untreated or uncontrolled hypertension is significantly higher in LMICs, mainly due to limited awareness and overburdened health systems [3].

Changing lifestyles (increased intake of processed food, alcohol and tobacco consumption, limited physical activity), rapid urbanization, aging populations and genetic factors all play a role in the increasing hypertension epidemic in LMICs [3,5-6]. There are other underlying factors that are not completely understood, including increasing prevalence of low birth weights or repeated episodes of certain infectious diseases [7,8].

'London School of Hygiene \& Tropical Medicine, Keppel Street, London, WC1E 7HT, UK

${ }^{2}$ Novartis Foundation, Novartis Campus Forum 1 - 3.93, CH-4056 Basel, Switzerland

${ }^{3}$ Imperial College London, The Hammersmith Hospital, Du Cane Road, London, W12 0NN, UK

${ }^{4}$ FHI360, 359 Blackwell Street, Suite 200, Durham, NC 27701, USA

5 International Development Research Centre (IDRC), 150 Kent Street, Ottawa, Ontario K1P OB2, Canada

${ }^{6}$ The University of the West Indies, Cave Hill Campus, Bridgetown BB11000, Barbados

${ }^{7}$ Centre for Chronic Conditions \& Injuries \& Public Health Foundation of India, Delhi NCR Plot No. 47, Sector 44, Institutional Area

Gurgaon - 122002, India

${ }^{8}$ The New York Academy of Medicine, 1216 Fifth Avenue, New York, NY 10029, USA

*Author for correspondence: pablo.perel@lshtm.ac.uk

\section{KEYWORDS}

- global health $\bullet$ healthcare

- healthcare telemedicine

- high blood pressure

- hypertension • low-

and middle-income

countries $\bullet$ management

- noncommunicable disease

Future
Medicine $\mathrm{fS}$ 
Addressing the needs of patients with chronic diseases is challenging for health systems already overwhelmed by infectious diseases and maternal-and-child health issues. There is an urgent need for innovation in healthcare delivery to tackle this dual burden. A recent study has shown it is possible to substantially improve hypertension control at a national level in only a few years [9]. Moreover, innovative care models for hypertension that successfully decrease the workload in health systems and improve patient outcomes could also create solutions for handling other noncommunicable diseases (NCD)s. This was the topic of the recent London Dialogue, cohosted by the Novartis Foundation and the London School of Hygiene \& Tropical Medicine Centre for Global NCDs on 1 December 2015.

Innovative delivery models for hypertension were presented from Kenya, Ghana, Barbados and India. The Sustainable model for Cardiovascular health by Adjusting Lifestyle and treatment with Economic perspective in settings of Urban Poverty (SCALE-UP) program in the urban slums of Nairobi, Kenya, demonstrated the importance of designing programs around patients' daily reality. An excellent set of interventions addressed gaps in the hypertension delivery chain (improving awareness, access to screening and quality treatment and promoting adherence). As part of the program, 50 community health workers were trained and equipped to conduct door-to-door hypertension screening, seven patient support groups were formed, six clinical officers and nurses were trained and a standardized clinical treatment guideline was developed. However, despite these interventions, the program demonstrated limited success; while three in four hypertensive patients were treated at least once, only one in three participants was retained in treatment for $>6$ months and $\mathrm{BP}$ control was achieved by just $6 \%$ of patients [10]. It highlighted that job-seeking takes priority over healthcare-seeking behavior in these settings. Alongside financial reasons, patients failed to adhere to treatment, mainly because of limited opportunities to attend health services during the day.

The Ghana Community Hypertension Improvement Project (ComHIP) has the patient at the center of intervention, using digital health to empower individuals in their own disease management, for example, through adherence support and lifestyle education. This model maximizes screening and care delivery outside the health system, by shifting these tasks to local pharmacies and community nurses, all connected by digital health to facilitate coordinated patient care. The model is based on a successful model in HIV, demonstrating how learnings from infectious diseases can be applied to NCDs.

India also provides innovative models for hypertension including the use of front-line healthcare workers to initiate structured behavior change (reduction of salt, harmful intake of alcohol, fruit and vegetable intake). One further example used innovative software to aid evaluation, risk stratification, drug management and lifestyle interventions for hypertension patients, and was associated with reductions in BP [11]. In Barbados, an overall lack of standardization in hypertension management (including poor adherence to hypertension protocols) was identified as a barrier to achieving successful hypertension control. The introduction and positive impact of new hypertension treatment guidelines showed that greater standardization to improve care is essential. Key to the success of this initiative was early identification and involvement of stakeholder groups.

However, healthcare alone is not sufficient to fully address hypertension, because many of the underlying factors fall beyond the scope of health systems. Tackling socioeconomic determinants could have much more impact and demands less effort from individuals [3]. There is consequently a need for a comprehensive approach to tackling hypertension in LMICs, based on cross-sectoral collaboration to bring together expertise and resources from private and public sectors.

Focusing on nontraditional health aspects has proven successful in high-income settings. One approach is the 'age-friendly city' example of New York, where older persons are asked to identify necessary changes across domains, such as transportation, housing, social participation, employment and health to improve their lives. This information is then used to shape actions by government agencies and private sector organizations. These principles are valid for LMICs as demonstrated by the WHO, highlighting the success, scalability and affordability of other population-based measures [12].

The hypertension challenge in the majority world has unprecedented scope. As the World Heart Federation's Roadmap demonstrates, proven cost-effective lifestyle and medical interventions to prevent and manage 
hypertension exist, though uptake is still unacceptably low and is reflected in important care gaps in LMICs [13]. Hypertension is one of the easiest NCDs to diagnose and treat, but there is a pressing need for new ideas and evidence to support the effectiveness of innovative models that are affordable, culturally acceptable and sustainable at scale. Future models must be designed around a thorough analysis of patients' reality. Optimizing the use of digital health to transform service delivery, reduce costs, broaden access and maximize outside screening and care opportunities could help alleviate the burden of NCDs on health systems. Innovative care models linked to changes in community conditions could address wider social determinants. However, this requires a holistic approach involving a variety of partners. If successful, treatment coverage of the condition is one of the candidate indicators to monitor universal health coverage and lessons from hypertension may prove applicable to innovative healthcare delivery models for other NCDs that are more difficult to diagnose and treat.

\section{Authorship declaration \\ The opinions expressed in this article were developed fol- lowing a Novartis Foundation event in London (UK) on 1 December 2015. All authors contributed equally to the article, and the work to develop the article was that of the contributing authors.}

\section{References}

1 Lim SS, Vos T, Flaxman AD et al. A comparative risk assessment of burden of disease and injury attributable to 67 risk factors and risk factor clusters in 21 regions, 1990-2010: a systematic analysis for the Global Burden of Disease Study 2010. Lancet 380(9859), 2224-2260 (2012).

2 Angell SY, De Cock KM, Frieden TR. A public health approach to global management of hypertension. Lancet 385(9970), 825-827 (2015).

3 WHO. A global brief on hypertension (2013). http://apps.who.int

4 Yusuf S, Rangarajan S, Teo K et al. Cardiovascular risk and events in 17 low-, middle-, and high-income countries. N. Engl. J. Med. 371, 818-827 (2014).

5 Larifla L, Beaney KE, Foucan L et al. Influence of genetic risk factors on coronary heart disease occurrence in Afro-Caribbeans. Can. J. Cardiol. doi: 10.1016/j.

Financial \& competing interests disclosure

The Novartis Foundation funded the travel and accommodation for the authors to meet at a Novartis Foundationsponsored dialogue event in London (UK) on 1 December 2015. The authors did not receive any other payment or fees. All authors have completed the Unified Competing Interest form at www.icmje.org/coi_disclosure.pdf (available on request from the corresponding author). Two of the authors (A Aerts, Head of the Novartis Foundation, and A Caldwell, Head Innovative Healthcare Delivery Solutions) are employed by the Novartis Foundation. P Piot has received grants from Gates Foundation, IMI, DFID and UNAIDS outside the submitted work and is a Board member for the Novartis Foundation. D Prabhakaran reports grants from Medtronic Foundation, outside the submitted work and has a patent copyright for HER and DSS issued. P Perel reports grants for the LSHTM (Com-HIP) programme from the Novartis Foundation during the conduct of the study. The authors have no other relevant affiliations or financial involvement with any organization or entity with a financial interest in or financial conflict with the subject matter or materials discussed in the manuscript apart from those disclosed.

Writing assistance was utilized in the production of this manuscript. The Novartis Foundation also funded a medical writer, T Ryder, to support the preparation of the final manuscript.

\section{Open access}

This work is licensed under the Attribution-Non CommercialNoDerivatives 4.0 Unported License. To view a copy of this license, visit http://creativecommons.org/licenses/ by-nc-nd/4.0/

cjca.2016.01.004 (2016) (Epub ahead of print).

6 Bayoglu B, Yuksel H, Cakmak HA et al. Polymorphisms in the long non-coding RNA CDKN2B-AS1 may contribute to higher systolic blood pressure levels in hypertensive patients. Clin. Biochem. doi: 10.1016/j. clinbiochem.2016.02.012 (2016) (Epub ahead of print).

7 Lackland DT, Egan BM, Ferguson PL. Low birth weight as a risk factor for hypertension. J. Clin. Hypertens. 5(2), 133-136 (2003).

8 Curry K, Lawson L. Links between infectious diseases and cardiovascular disease: a growing body of evidence. J. Nurse Pract. 5(10), 733-741 (2009).

9 Falaschetti E, Mindell J, Knott C et al. Hypertension management in England: a serial cross-sectional study from 1994 to 2011. Lancet 383(9932), 1912-1919 (2014).

10 Oti SO, van de Vijver SJ, Kyobutungi C et al. A community-based intervention for primary prevention of cardiovascular diseases in the slums of Nairobi: the SCALE UP study protocol for a prospective quasi-experimental community-based trial. Trials 14, 409 (2013).

11 Anchala R, Kaptoge S, Pant H et al. Evaluation of effectiveness and cost-effectiveness of a clinical decision support system in managing hypertension in resource constrained primary health care settings: results from a cluster randomized trial. J. Am. Heart Assoc. 4(1), e00121 (2015).

12 World Health Organization. From burden to 'best buys': reducing the economic impact of non-communicable diseases in low- and middle-income countries (2011). www.who.int

13 Adler AJ, Prabhakaran D, Bovet P et al. Reducing cardiovascular mortality through prevention and management of raised blood pressure: a World Heart Federation Roadmap. Glob. Heart 10(2), 111-122 (2015). 